\title{
Information-Based Numerical Distances between Equilibrium and Non-Equilibrium States
}

\author{
Angel Ricardo Plastino1,2, Gustvo Luis Ferri' ${ }^{3}$, Mario Carlos Rocca ${ }^{2,4,5}$, Angelo Plastino ${ }^{2,4,6}$ \\ ${ }^{1}$ CeBio y Departamento de Ciencias Básicas, Universidad Nacional del Noroeste de la Prov. de Buenos Aires, Junin, Argentina \\ ${ }^{2}$ Argentina's National Research Council (CONICET), La Plata, Argentina \\ ${ }^{3}$ Departamento de Física, Facultad de Ciencias Exactas y Naturales, Universidad Nacional de La Pampa, Junin, Argentina \\ ${ }^{4}$ Departamento de Física, Universidad Nacional de La Plata, La Plata, Argentina \\ ${ }^{5}$ Departamento de Matemática, Universidad Nacional de La Plata, La Plata, Argentina \\ ${ }^{6}$ SThAR - EPFL, Lausanne, Switzerland \\ Email: angeloplastino@gmail.com
}

How to cite this paper: Plastino, A.R., Ferri, G.L., Rocca, M.C. and Plastino, A. (2020) Information-Based Numerical Distances between Equilibrium and Non-Equilibrium States. Journal of Modern Physics, 11, 10311043.

https://doi.org/10.4236/jmp.2020.117065

Received: June 20, 2020

Accepted: July 11, 2020

Published: July 14, 2020

Copyright $\odot 2020$ by author(s) and Scientific Research Publishing Inc. This work is licensed under the Creative Commons Attribution International License (CC BY 4.0).

http://creativecommons.org/licenses/by/4.0/

\begin{abstract}
We consider a typical master equation describing thermal time-evolution. In parallel, we also consider a quasi static canonical description of the same problem. We are able to devise a way of numerically comparing these two treatments and concoct a distance-measure between them. In this way, one is in a position to know how far or close equilibrium and off-equilibrium can get. The first, rather surprising observation, is that our systems lose structural details as $N$ grows. Also, the time-evolution of the distance between the two pertinent probability distributions is quite sensitive to the heating-cooling process.
\end{abstract}

\section{Keywords}

Information Theory, Master Equation, Thermal Time-Evolution, Equilibrium-Off Equilibrium Distances

\section{Introduction}

\subsection{Preliminaries}

Researchers often appeal to master equations (ME) to obtain an equation of motion for the reduced density operator. Or for the probability distribution (PD) of a subsystem of interest $A$ in interaction with (a usually much larger) subsystem B (heath bath, for instance). The key issue is that our system of interest A is in a 
situation of off-equilibrium. The literature on the subject is really enormous (Google Scholar returns lists around 30,000 links). Thus, we content ourselves with citing [1] and references therein. The issue at hand is how to extract relevant information on system A from the pertinent von Neumann equation. Consequently, our aim is to derive the time evolution of the $\mathrm{PD}$ for the entire system $\mathrm{A}+\mathrm{B}$, in a way which guarantees that normalization (amongst other properties) is preserved at any time $t$. The solution to this problem is found in the so-called master equation (ME) technique [1]. A popular, but not rigorous ME-approach can be used in the case of physical situations for which interacting systems A and $\mathrm{B}$ are known and well-defined so that one constructs the corresponding ME-equation of motion for the PD [1]. A beautiful instantiation of the ME-procedure is presented by Takada, Conradt, and Richet (TCR) in [2], for example, we will follow here without further ado.

\subsection{TCR Main Ideas}

TCR consider a two-level ( 1 and 2) model (system A) in contact with a reservoir $\mathrm{B}$ of temperature $T$. If the population of the excited state is computed, then that of the ground state becomes automatically fixed. The transition rate is the crucial parameter governing the degree of non-equilibrium. Denote by $p_{1}$ and $p_{2}$ the concomitant occupation probabilities of the lower and upper wells, respectively.

TCR imagine a heating and cooling process in which the reservoir's temperature is a function of time. $T$ first grows, attains a maximum value, and then decreases. One can interpret this scenario as that of a particle moving in an asymmetric double-well potential. Site 1 is the bottom of the first well, whose energy is $E_{1}$. Likewise, site 2 is the bottom of the second well, at a higher energy $E_{2}$. Then, $E_{1}$ is the potential energy barrier to be overcome between states 1 and 2 . System A subsequently evolves with a concomitant energy decrease to $E_{2}$, leading to state 2. See Figure 1. TCR write the associated master equation as

$$
\mathrm{d} p_{1} / \mathrm{d} t=-a_{1} p_{1}+a_{2} p_{2} ; p_{1}+p_{2}=1,
$$

with

$$
a_{i}=\exp \left[-E_{i} / k_{b} T\right], i=1,2, k_{B}=\text { Boltzmann's constant. }
$$

\subsection{Present Goal}

Inspired by [2], we wish to address here a different problem. We will tackle a quantum many-body system of interacting fermions, advanced in [3], for which the interaction is ruled by an SU2 algebra. The system is heated and cooled as depicted in Figure 1, and we will deal with a master coupled system of $N$ equations (not just two as in [2]). The thermal process will compete with the fermion-fermion interaction's effects. A canonical ensemble treatment of this model is reported in [4]. In parallel, for every different temperature $T$, we consider a fictitious coupled system-reservoir in thermal equilibrium at such $T$ (quasi-static approximation (qsa). We will be able to devise, via Information Theory quantifiers, several notions of distance between the "master equation probability distribution" 


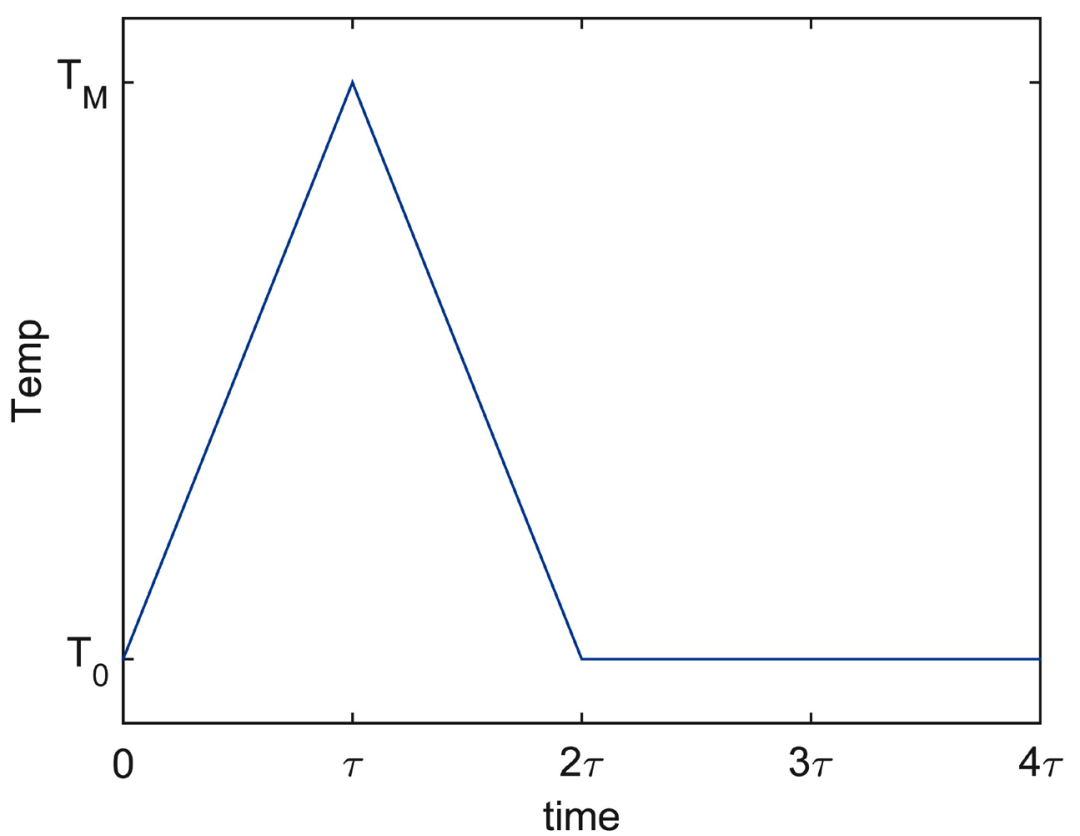

Figure 1. Time evolution of the heath-bath temperature $T_{0}<T<T_{M}$ between a time $0<t<2 \tau . T=T_{0}$ for $T<2 \tau$.

(MEPD) and the "qsa" PD, which of course, will yield numerical values that measure how far or close the two treatments are.

Epistemology tells us that classification is an essential feature of scientific endeavor [5]. For such a task we need numbers, and the distances that we are looking for provide such numbers, perhaps for the first time ever in the present context.

\subsection{Paper's Organization}

Sections 2 and 3 describe appropriate details of our exactly solvable quantum many-body system, that will serve as a laboratory to test our ideas of thermal distance between off equilibrium and equilibrium distances (OEED). Section 4 illustrates about the notion of statistical complexity. Section 6 is of the essence, as it presents our main ideas: that of a) a master equation and b) of quantifiers of OEED. The main results are exhibited and discussed in Section 7 and, finally, some conclusions are drawn in Section 8.

\section{M-Fermions' Exactly Solvable Model}

The model advanced in Ref. [3] and further discussed in Ref. [4], considers $M$ fermions distributed amongst ( $2 M$ )-fold degenerate single-particle (sp) levels, separated by a sp energy gap $\epsilon$. Two quantum numbers $\mu$ and $p$ are attached to a generic single particle state. The first adopts the values $\mu=-1$ (lower level) and $\mu=+1$ (upper level). $p$, usually called quasi-spin or pseudo spin, singles out a state within the $M$-fold degeneracy. The couple $p, \mu$ can also be viewed as a "site" that is either occupied or empty. One has 


$$
M=2 J,
$$

where $J$ denotes an "angular momentum". One introduces now the quasi-spin operators

$$
\begin{gathered}
\hat{J}_{+}=\sum_{p} C_{p,+}^{\dagger} C_{p,-}, \\
\hat{J}_{-}=\sum_{p} C_{p,-}^{\dagger} C_{p,+}, \\
\hat{J}_{z}=\sum_{p, \mu} \mu C_{p, \mu}^{\dagger} C_{p, \mu}, \\
\hat{J}^{2}=\hat{J}_{z}^{2}+\frac{1}{2}\left(\hat{J}_{+} \hat{J}_{-}+\hat{J}_{-} \hat{J}_{+}\right),
\end{gathered}
$$

the eigenvalues of $\hat{J}^{2}$ being of the form $J(J+1)$. The Hamiltonian of reference [3], a spin-flip one, reads

$$
\hat{H}=\epsilon \hat{J}_{z}-V_{s}\left(\frac{1}{2}\left(\hat{J}_{+} \hat{J}_{-}+\hat{J}_{-} \hat{J}_{+}\right)-\hat{J}\right),
$$

or, with $V=V_{s} / \epsilon \quad$ (equivalently, take $\epsilon=1$ ),

$$
\hat{H}=\hat{J}_{z}-V\left(\frac{1}{2}\left(\hat{J}_{+} \hat{J}_{-}+\hat{J}_{-} \hat{J}_{+}\right)-\hat{J}\right),
$$

so that the unperturbed ground state (ugs) $(V=0)$ becomes, according to Equation (3),

$$
\left|J, J_{z}\right\rangle=|J,-M / 2\rangle
$$

whose energy is

$$
E_{o}=-M / 2 .
$$

Doubly occupied $p$-sites are not allowed for. The Hamiltonian commutes with $\hat{J}^{2}$ and $\hat{J}_{z}$. This entails that the exact solution will belong to the $J$-multiplet of the unperturbed ground state. The multiplet's states are denoted as $|J, m\rangle$, and one of them will minimize the energy. The associated $m$ value of this state will depend upon the coupling strength $V$ of the interaction. As we just mentioned, for the ugs one has $m=-J=-M / 2$. Evidently, the interaction-operator $\left(\hat{J}_{+} \hat{J}_{-}+\hat{J}_{-} \hat{J}_{+}\right)$is a quasi-spin flipping operator. Thus, this operator becomes the more "effective" the more balanced the populations of the two-levels become.

\section{$T=0$-Phase Transitions}

As $V$ grows from zero, the ugs energy $E_{o}$ is not immediately affected. It conserves its value till a critical $V$-specific value is attained, of $1 /(M-1)$. At this juncture, the interacting gs suddenly turns out to be $|J,-M / 2+1\rangle$. If $V$ continues its growth, new phase transitions (PT) take place. The PT between $J_{z}=-k$ and $J_{z}=-k+1$ ensues at $V=1 /(2 k-1)$. The successive PT's processes ends up when we attain either $J_{z}=0 \quad\left(V_{\text {crit }}=1\right.$ for integer $)$, or $J_{z}=-1 / 2 \quad\left(V_{\text {crit }}=1 / 2\right.$ for odd $)$. Thus, at such juncture one has, independently of $J[3]$ : 


$$
V_{\text {crit }}=1 / 2 \text { for half-integer } J \text { or } V_{\text {crit }}=1 \text { for integer } J \text {. }
$$

\section{Model's Treatment at Finite Temperatures $T$}

To repeat: double occupancy of a $p$-site is not permitted. Thus, the Hamiltonian matrix' size is $(2 J+1) \times(2 J+1)$. The only way to get different $\rho_{\mathrm{S}}$ is to have double occupancy [4]. Following this last reference, the $J=N / 2$ multiplet is the only one we need to consider.

The free energy $F$ and the partition function $Z$, with $\beta$ the inverse temperature, and $k_{B}=1$ (Boltzmann) are given by

$$
F=-T \ln Z=-T \ln \text { Trace }(\exp (-\beta \hat{H})) .
$$

In the Trace we sum over the $J_{z}$ quantum number $m$. As $H$ commutes with both $J$ and $J_{z}$ one finds

$$
Z=\sum_{m=-J}^{m=J} \exp \left(-\beta E_{m}\right)
$$

where the energy $E_{m}$ are

$$
E_{m}=m-V\left(J(J+1)-m^{2}-J\right) .
$$

Our all important pertinent probabilities $P_{m}$ are [4]

$$
P_{m}=\frac{\exp \left(-\beta E_{m}\right)}{Z},
$$

for all $m=-J,-J+1, \cdots, J-1, J$. The entropy $S$ is

$$
S=-\sum_{m=-J}^{m=J} P_{m} \ln P_{m} .
$$

\section{Meaning of the Statistical Complexity Measure}

Sometimes one wishes to grab hold of a system's correlation structures just as entropy grasps disorder. Why? Because such correlations strongly influence the main features of the prevailing PD describing physical processes. It is obvious that the opposite extremes of perfect order and maximal randomness do not manifest notable structural correlations [6]. In between these two instances, a wide range of structural degrees (SD) usually exist, that are in turn reflected by the traits of the prevailing PD $P$.

The authors of Ref. [6], invented a quite adequate functional $F[P]$ that can apprehend correlations just as Shannon's entropy encapsulates randomness, which indeed constituted an important breakthrough. Their ideas were conceptualized via the definition of López-Ruiz, Mancini, and Calbet (LMC) [6] of what became called the statistical complexity $C$.

LMC $C$ individualized and quantified the respective contributions of entropy and structure. The last one was described by a quantity called disequilibrium $D$. Their concept of statistical complexity $C$ was widely accepted (for a sample see, for instance, Refs. [6]-[27]). $C$ vanishes in the two situations of perfect order and maximum disorder, being defined as the product of Shannon's entropy $S$ and the 
disequilibrium $D$. More specifically, the latter is a measure in probability space of the distance measure the prevailing $\mathrm{PD}$ and the uniform PD, so that one writes

$$
C=S D \text {, }
$$

with (see (17)), in our case, the uniform probabilities are $P^{(u)}=1 /(2 J+1)$ for all $m$ between $-J$ and $J$, so that, the LMC disequilibrium is

$$
D=\sum_{m=-J}^{m=J}\left(P_{m}-P^{(u)}\right)^{2},
$$

while

$$
S=-\sum_{m=-J}^{m=J} P_{m} \ln P_{m} .
$$

For details, properties, and others applications of $C$, see Refs. [6].

\section{Master Equations for $N$-Levels Systems}

We consider that our system is at the equilibrium temperature $T_{0}$ at $t=0$. Then he system is heated and reaches a temperature $T_{M}$ at $t=\tau$. At this stage, the systems cools-off, reaching a temperature $T_{f}=T_{0}$ at time $t=2 \tau$. This is the temperature of the heat-reservoir at all later times.

Inspired by the 2-level systems treatment of [2], we tackle now a system of $N$ levels $l_{i}(i=0,1,2, \cdots)$, with level-energies $E_{0}, E_{!}$, etc., and selections rules that allow for only certain kinds of transitions. In the usual parlance of quantum many body theories, we permit only transitions from a state with $k$-particleholes ( $\mathrm{p}-\mathrm{h})$ to ones with either $k+1$ or $k-1 \mathrm{p}$-h, so that

$$
\frac{\mathrm{d} p_{n}}{\mathrm{~d} t}=p_{n-1} \exp \left(-\beta E_{n}\right)-p_{n} \exp \left(-\beta E_{n-1}\right),
$$

and we follow in this way till we face, for the last 3 steps,

$$
\begin{aligned}
\frac{\mathrm{d} p_{2}}{\mathrm{~d} t}=p_{1} \exp \left(-\beta E_{2}\right)-p_{2} \exp \left(-\beta E_{1}\right), \\
\frac{\mathrm{d} p_{1}}{\mathrm{~d} t}=p_{0} \exp \left(-\beta E_{1}\right)-p_{1} \exp \left(-\beta E_{0}\right), \\
\frac{\mathrm{d} p_{0}}{\mathrm{~d} t}=-\left[p_{n-1} \exp \left(-\beta E_{n}\right)-p_{n} \exp \left(-\beta E_{n-1}\right)\right]-\cdots \\
-\left[p_{0} \exp \left(-\beta E_{1}\right)-p_{1} \exp \left(-\beta E_{0}\right)\right] .
\end{aligned}
$$

The last equation guarantees normalization of probabilities.

The initial conditions are $p_{i}(0)=\exp \left(-E_{i} / k T\right) / Z$ for all $i$.

$\beta$ depends upon time, as described at the beginning of this Section and we work with

$$
\beta(t)=1 / T(t),
$$

and

$$
E_{j}=j ; \text { all } j \text {. }
$$


If $\beta$ were constant, then the time-dependent probabilities $p_{i}(t)$ would relax to stationary Gibbs-distributions.

The numerical calculations consider the cases of $N=3,4,5,6$, corresponding respectively to $J=1,3 / 2,2,5 / 2$ for our model above.

\section{Distance Quantifiers}

We deal with the results of our master equation (ME) above, that are to be compared to the quasi-static (st) results that arise out of considering always Boltzmann-Gibbs equilibrium-situations at the temperature $T(t)$ for all $t$. As distance quantifiers we will employ

- The probabilities' differences 1) Global

$$
\begin{aligned}
& D_{P}=\sum_{m}\left[P_{m}^{M E}(t)-P_{m}^{s t}(t)\right]^{2} /\left(P_{m}^{M E}(t)\right)^{2}, \text { and 2) Individual } \\
& d_{P}^{m}=\left[P_{m}^{M E}(t)-P_{m}^{s t}(t)\right]^{2} /\left(P_{m}^{M E}(t)\right)^{2},
\end{aligned}
$$

- the entropy $S$,

- the free energy $F$,

- the mean energy $U$, and

- the statistical complexity $C$.

If we generically call $Q$ to any of these quantifiers, the distances are of the form

$$
\begin{gathered}
d_{Q}(t)=\frac{\left[Q_{M E}(t)-Q_{s t}(t)\right]^{2}}{Q_{M E}(t)^{2}}, \\
D_{Q}=\int_{0}^{20 \tau} \mathrm{d} t d_{Q}(t),
\end{gathered}
$$

where, obviously, the sub-index ME (or just " $M$ ") refers to master equation's results, and the sub-index "st" (or just "s") to quasi-static ones.

We expect the distances to be sensitive to

1) Changes in the behavior of $T$ with $t$ and

2) Structural system's changes with the coupling constant $V$,

3) The speed of the heating-up and cooling-off process, regulated by the parameter $\tau$. The shorter $\tau$, the faster the speed.

\section{Distances' Results}

Note that, with reference to our model above, we have $N=2 J+1$. Figure 2 refers to $N=3$ and the individual probabilities-distance $D_{P}^{m}$ vs. $t$. It is clearly seen that there is much sensitivity to the details of the heating process. Figure 3 refers also to $N=3$ but with the global $D_{P}$ vs. $V$. Here the ensuing picture is of a more complex nature. There is a system's phase transition (PT) at $V=1$, and our distance tends to decrease as we approach the PT. Thus we see that $D_{P}$ is sensitive to the internal dynamics of the system. This is no trivial issue. Why should the OE-equilibrium distance behave in such a manner? This is a new fact discovered here, as far as we know. But the picture becomes even more complex when we consider the speed of the heating process. If it is large enough, the distance OE-E grows again with $V$ after the PT, but if it is low enough, it continues 


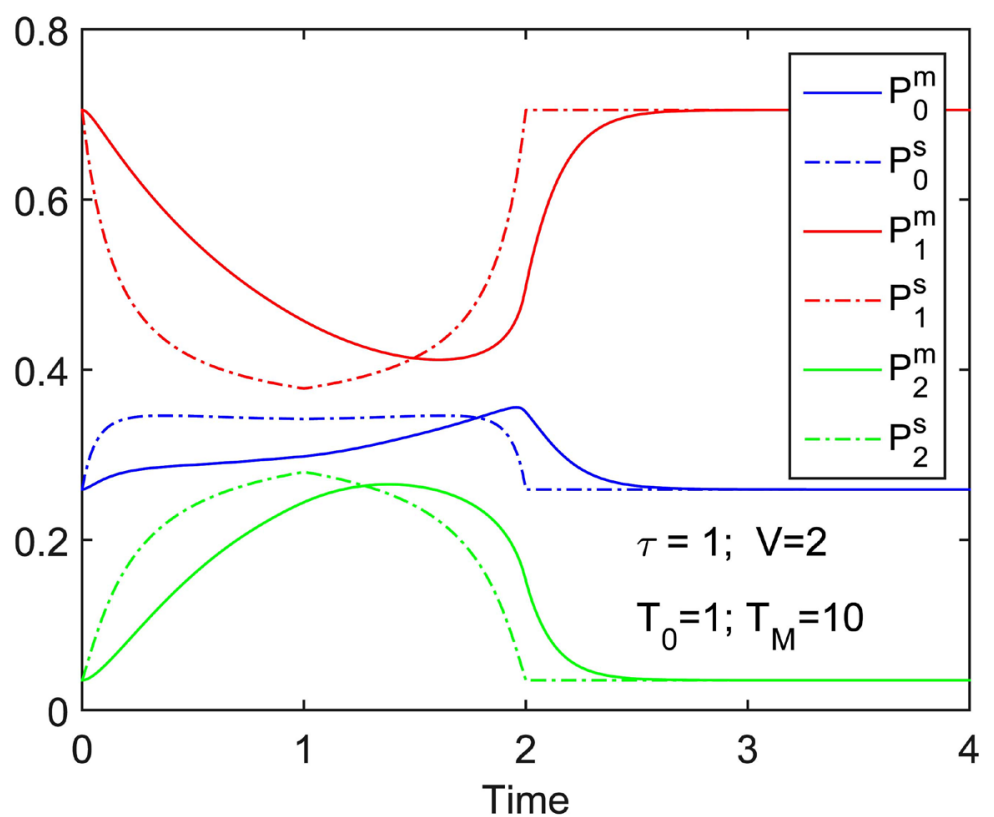

Figure 2. The distance $D_{O E_{E}}$ (see text) off equilibrium-equilibrium for different $\tau$ values.

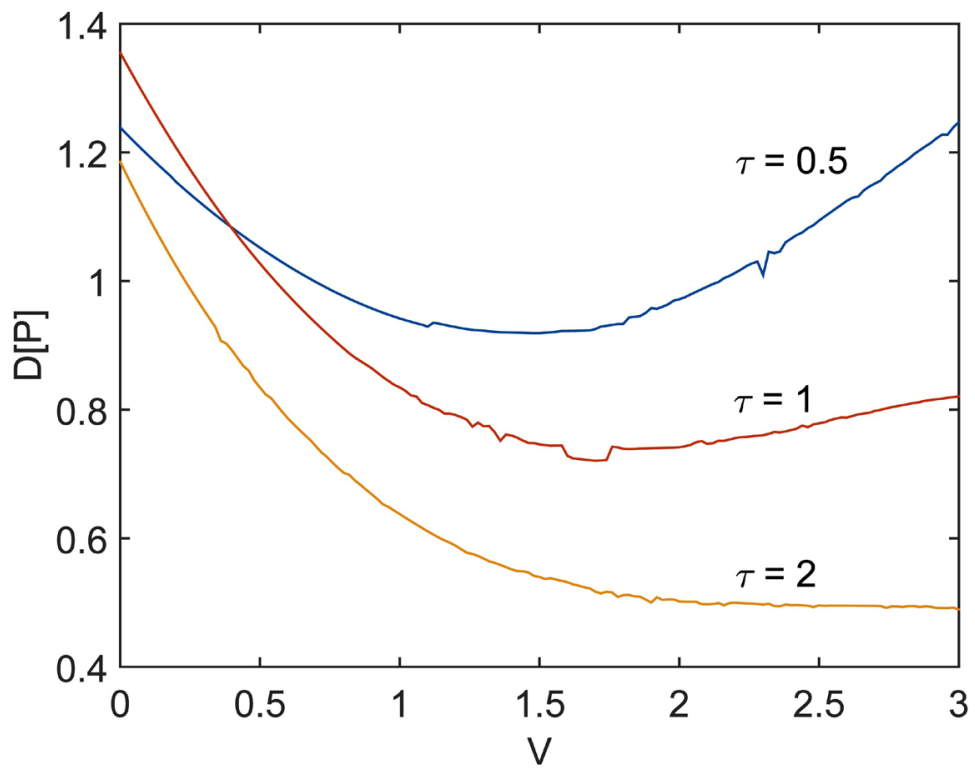

Figure 3. $N=3$ : the distance $D_{O E_{E}}$ vs. the coupling constant $V$ for several $\tau$ values (see text).

decreasing as $V$ grows. We conclude that the distance quantifiers is sensitive both to the system's internal dynamics and to the details of the heating process.

Starting now with $N=4$ we pass to watch in Figure 4 the performance with time of the other quantifiers, of thermal origin, namely $S, F, U$, and $C$. As compared with the probabilities-distance, the thermal-distance (TD) is much more sensitive to the details of the heating process. When it finishes, at $t=2 \tau$, the four different TD vanish. We pass now to Figure 5 so as to analyze, for $N=3$, 


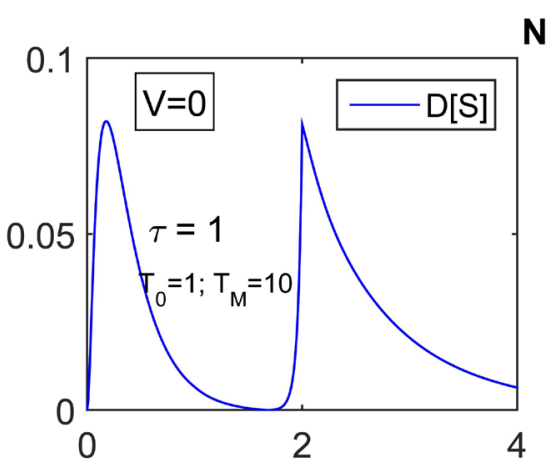

$\mathbf{N}=4$
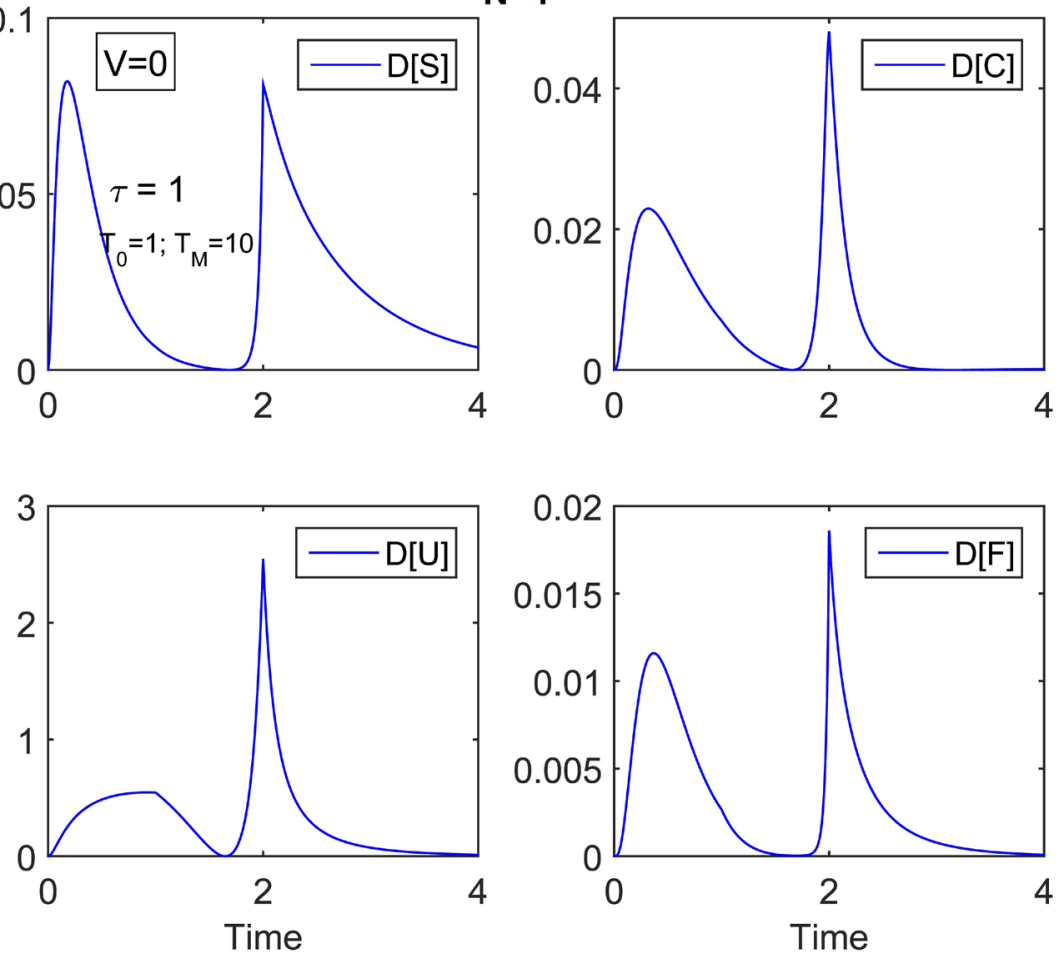

Figure 4. The four thermal distances vs. time for $N=4$ (see text).
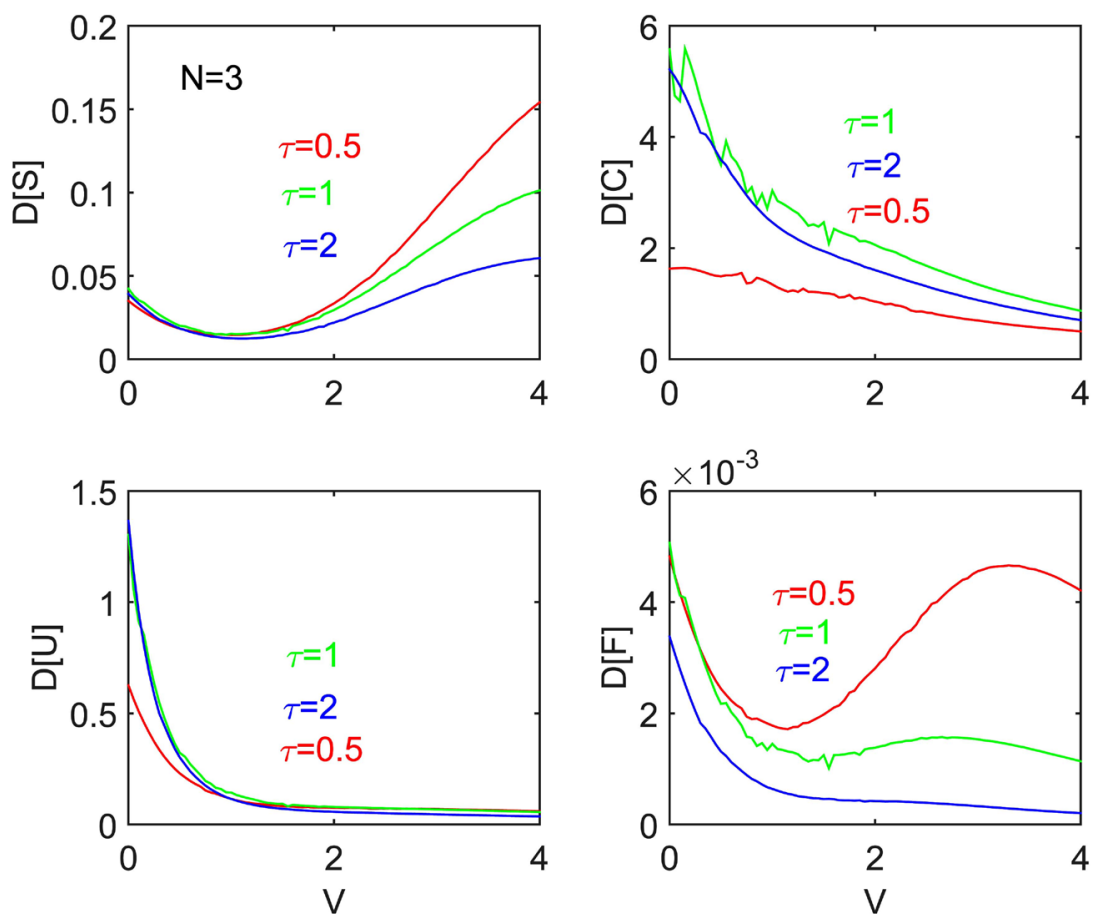

Figure 5. $N=3$ : the four thermal distances vs. the coupling constant $V$ for several $\tau$ values (see text).

the behavior of the four thermal distances. We see that each of them behaves in a distinctive way, that forces individual consideration. 
- $S$-distance: it diminishes as $V$ grows from zero till reaching the phase transition (PT). After wards it grows again as $V$ continues increasing. At the PT the distance is minimal. The after-PT grows is the more pronounced the speedier the heating process (HP). Even after it has finished, $S$ seems to keep memory of it, since its growth differentiates amongst the different $\tau$ 's.

- F-distance: It senses the PT if the HT is fast. Otherwise, it does not. It clearly is able fo distinguish amongst the different $\tau$ 's.

- $U$-distance: tends to vanish as $V$ grows. It is rather indifferent both to the $\tau$ -value and to the PT.

- $C$-distance: this is supposed to be the most sophisticated statistical of our four tools. Here it only tells us that the difference between the off-equilibrium complexity and the equilibrium one tend to become identical as the coupling constant grows. In other words, if the system is tightly bound, whether one heats it up or not becomes less and less relevant as the bonding augments, which sounds reasonable.

Figure 6 is identical to Figure 5, except for the fact that $N$ is larger and equals 5. There are two, not just one, PTs now, both for $V<1$, that do not seem to leave any trace in the graphs. The differences between what the different thermal indicators say is smaller here. All of them indicate that if the system is tightly bound, whether one heats it up or not becomes less and less relevant as the bonding augments, as we saw above for $N=3$, but only in the $C$-case. Here, once again, the statistical complexity appears to be the mpst sensitive of the indicators, as the $C$-distance is the only one of the four here used able to detect differences amongst the distinct $\tau$ values. These conclusions are reinforced if
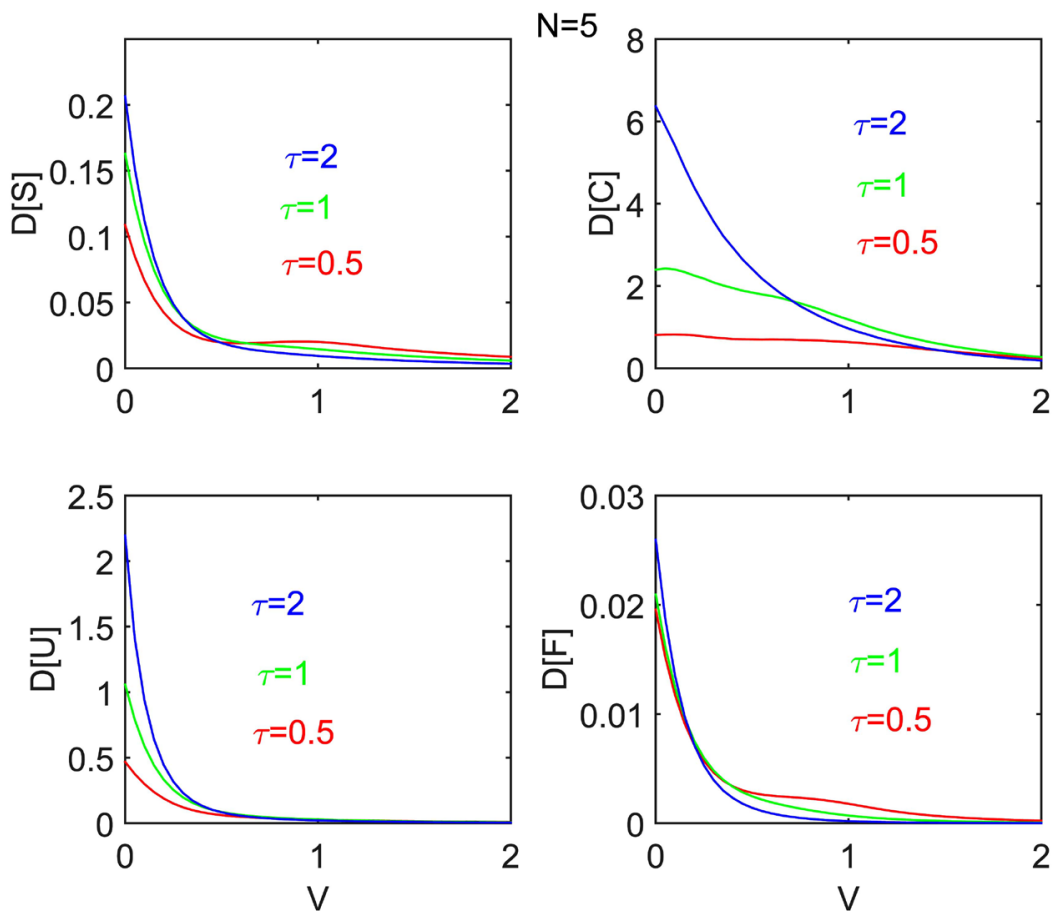

Figure 6. $N=5$ : same details as in Figure 5, but for a larger $N$-value (see text). 

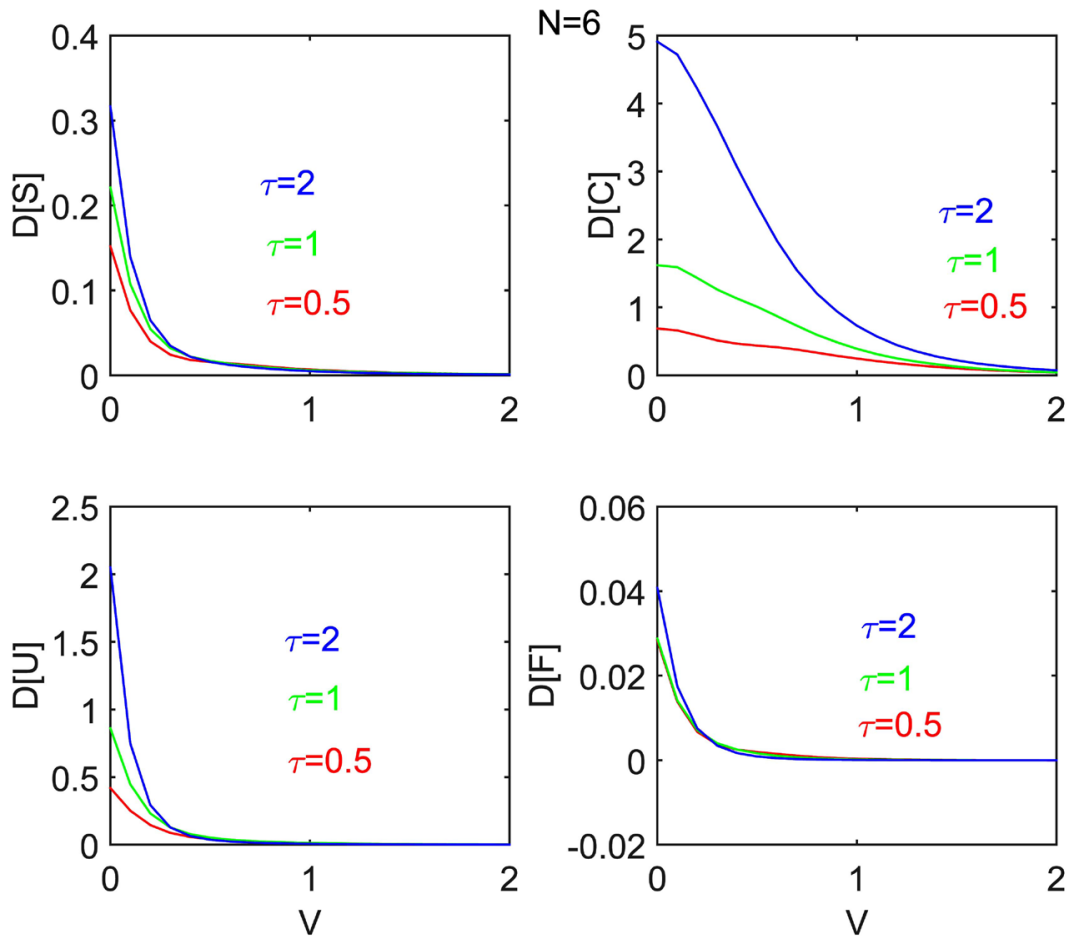

Figure 7. $N=6$ : same details as in Figure 5, but for a larger $N=6$-value (see text).

we confront now the case $N=6$. Emphasize that $C$ is undoubtedly the most sensitive quantifier (Figure 7).

\section{Conclusions}

- The first, rather surprising observation, is that our systems lose structural details as $N$ grows.

- The time-evolution of the distance between the two pertinent probability distributions is quite sensitive to the heating-cooling process.

- The shorter the heating-coolong period, the more sensitive the probabilities-distance quantifier becomes to the internal systems' structure, as revealed by the ground-state phase transition.

- The four thermal distances time-evolutions are also quite sensitive to the heating-cooling process.

- The four thermal distances evolutions with a growing coupling constant are quite sensitive to the internal dynamics and to $g$ the heating-cooling details for $N=3$.

- This sensitivity is gradually lost as $N$ grows.

- For $N \geq 4$, the four thermal distances evolutions with a growing coupling constant rapidly vanish. The strongly interacting systems seem no to care whether it is heated or cooled.

- This entails that, the larger the coupling constant $V$, the more rapidly equilibrium is attained, as evidences by the diminution of the values of our thermal distance quantifiers. However, $C$ is the most sensitive of the four indicators. 


\section{Conflicts of Interest}

The authors declare no conflicts of interest regarding the publication of this paper.

\section{References}

[1] Kryszewski, S. and Czechowska-Kryszk, J. (2008) Master Equation-Tutorial Approach.

[2] Takada, A., Conradt, R. and Richet, P. (2013) Journal of Non-Crystalline Solids, 360, 13. https://doi.org/10.1016/j.jnoncrysol.2012.10.002

[3] Plastino, A. and Moszkowski, S.A. (1978) Il Nuovo Cimento, 47, 470. https://doi.org/10.1007/BF02896236

[4] Pennini, F. and Plastino, A. (2018) Physica A, 491, 305. https://doi.org/10.1016/j.physa.2017.09.041

[5] Ziman, J. (2002) Real Science. Cambridge Univ. Press, Cambridge.

[6] López-Ruiz, F.R., Mancini, H. and Calbet, X. (1995) Physics Letters A, 209, 321. https://doi.org/10.1016/0375-9601(95)00867-5

[7] Feldman, D.P. and Crutchfield, J.P. (1998) Physics Letters A, 238, 244. https://doi.org/10.1016/S0375-9601(97)00855-4

[8] Martin, M.T., Plastino, A. and Rosso, O.A. (2003) Physics Letters A, 311, 126. https://doi.org/10.1016/S0375-9601(03)00491-2

[9] Kowalski, A., Martin, M.T., Plastino, A., Proto, A. and Rosso, O.A. (2003) Physics Letters $A, 311,180$. https://doi.org/10.1016/S0375-9601(03)00470-5

[10] Ribeiro, H.V., Zunino, L., Lenzi, E.K., Santoro, P.A. and Mendes, R.S. (2012) PLoS ONE, 7, e40689. https://doi.org/10.1371/journal.pone.0040689

[11] Ribeiro, H.V., Zunino, L., Mendes, R.S. and Lenzi, E.K. (2012) Physica A, 391, 2421. https://doi.org/10.1016/j.physa.2011.12.009

[12] Manzano, D. (2012) Physica A, 391, 6238. https://doi.org/10.1016/j.physa.2012.06.058

[13] Borgoo, A., Geerlings, P. and Sen, K.D. (2011) Physics Letters A, 375, 3829. https://doi.org/10.1016/j.physleta.2011.09.031

[14] Dehesa, J.S., López-Rosa, S.S. and Manzano, D. (2009) The European Physical Journal D, 55, 539. https://doi.org/10.1140/epjd/e2009-00251-1

[15] Esquivel, R.O., Molina-Espritu, M., Angulo, J.C., Antolín, J., Flores-Gallegos, N. and Dehesa, J.S. (2011) Molecular Physics, 109, 2353.

https://doi.org/10.1080/00268976.2011.607780

[16] López-Ruiz, R. (2013) Concepts and Recent Advances in Generalized Information Measures and Statistics. Bentham Science Publishers, Sharjah, 147-168. https://doi.org/10.2174/9781608057603113010012

[17] Sen, K.D. (2020) Quantum Reports, 20, 313.

[18] Mitchell, M. (2009) Complexity: A Guided Tour. Oxford University Press, Oxford.

[19] Martin, M.T., Plastino, A. and Rosso, O.A. (2006) Physica A, 368, 439. https://doi.org/10.1016/j.physa.2005.11.053

[20] Pennini, F. and Plastino, A. (2017) Physics Letters A, 381, 212. https://doi.org/10.1016/j.physleta.2016.11.023

[21] Pennini, F. and Plastino, A. (2018) Physica A, 506, 828. 
https://doi.org/10.1016/j.physa.2018.05.003

[22] Pennini, F. and Plastino, A. (2017) Physica A, 488, 85. https://doi.org/10.1016/j.physa.2017.07.005

[23] Pennini, F. and Plastino, A. (2019) Entropy, 21, 558. https://doi.org/10.3390/e21060558

[24] Branada, R., Pennini, F. and Plastino, A. (2018) Physica A, 511, 18. https://doi.org/10.1016/j.physa.2018.07.037

[25] Pennini, F. and Plastino, A. (2018) Physica, 491, 305. https://doi.org/10.1016/j.physa.2017.09.041

[26] Pennini, F. and Plastino, A. (2017) Physics Letters A, 381, 3849. https://doi.org/10.1016/j.physleta.2017.10.025

[27] Anteneodo, C. and Plastino, A.R. (1996) Physics Letters A, 223, 348. https://doi.org/10.1016/S0375-9601(96)00756-6 